Bárbara Annabelle Pesántez-Célleri; Juan Carlos Erazo-Álvarez; Cecilia Ivonne Narváez-Zurita; Verónica PaulinaMoreno

http://dx.doi.org/10.35381/r.k.v5i10.717

\title{
Estrategias de emprendimiento para el turismo comunitario
}

\section{Entrepreneurship strategies for community tourism}

\author{
Bárbara Annabelle Pesántez-Célleri \\ barbara.pesantez@psg.ucacue.edu.ec \\ Universidad Católica de Cuenca, Cuenca \\ Ecuador \\ https://orcid.org/0000-0002-6337-905X \\ Juan Carlos Erazo-Álvarez \\ jcerazo@ucacue.edu.ec \\ Universidad Católica de Cuenca, Cuenca \\ Ecuador \\ https://orcid.org/0000-0001-6480-2270 \\ Cecilia Ivonne Narváez-Zurita \\ inarvaez@ucacue.edu.ec \\ Universidad Católica de Cuenca, Cuenca \\ Ecuador \\ https://orcid.org/0000-0002-7437-9880 \\ Verónica Paulina Moreno \\ verónica.moreno@ucacue.edu.ec \\ Universidad Católica de Cuenca, Cuenca \\ Ecuador \\ https://orcid.org/0000-0003-1517-6124
}

Recibido: 20 de marzo de 2020

Revisado: 11 de abril de 2020

Aprobado: 07 de mayo de 2020

Publicado: 19 de mayo de 2020

\section{RESUMEN}

El objetivo de la investigación fue diseñar estrategias de emprendimiento de turismo comunitario para fortalecer el desarrollo socio-económico de los habitantes de Playas Villamil. La metodología aplicada fue de tipo descriptiva, no experimental. De los resultados obtenidos, el turismo comunitario se desarrolla de manera empírica por lo que se busca potenciar sobre emprendimiento estratégico en las comunidades del Cantón. Es necesario considerar el desarrollo de emprendimientos estratégicos en las comunidades del Cantón Playas Villamil para fortalecer su nivel socioeconómico con la innovación de productos y servicios atractivos al turista. 
Bárbara Annabelle Pesántez-Célleri; Juan Carlos Erazo-Álvarez; Cecilia Ivonne Narváez-Zurita; Verónica PaulinaMoreno

Descriptores: Desarrollo comunitario; estrategia de desarrollo; turismo; economía informal. (Palabras tomadas del Tesauro UNESCO).

\begin{abstract}
The objective of the research was to design community tourism entrepreneurship strategies to strengthen the socio-economic development of Villamil beach's inhabitants. The applied methodology was descriptive and non-experimental. From the results obtained, community tourism is developed empirically so it seeks to promote strategic entrepreneurship in the Canton society. It is necessary to consider the development of strategic ventures in Villamil beaches of the Canton population to enhance their socio-economic level with the innovation of products and services attractive to tourists.
\end{abstract}

Descriptors: Community development; development strategy; tourism; informal economy. (Words taken from the UNESCO Thesaurus).

\title{
INTRODUCCIÓN
}

El Ecuador en su desarrollo socio-económico según (Instituto nacional de estadística y censos, 2019) en el mes de Diciembre del año 2019, el nivel de pobreza en el ámbito nacional se ubicó en $25.0 \%$, y la pobreza extrema se situó en $8.9 \%$, entendiéndose a una persona pobre por ingresos mensuales menores a USD 84,82 y si percibe menos de USD 47,80 es considerado pobreza extrema, y específicamente en las principales ciudades del Ecuador la pobreza en Guayaquil cuenta con $11.20 \%$, en Quito con $8.2 \%$ y Cuenca con $4.10 \%$, y con respecto a la pobreza extrema Guayaquil consta con $1.4 \%$, Quito con $2.8 \%$ y Cuenca con $0.6 \%$.

En la provincia del Guayas en especial y en las grandes ciudades con mayor movimiento y a nivel de empresa siendo fuente de desarrollo productivo con sus capitales, en esta provincia constan la mayoría de negocios con un $19.10 \%$ en comparación de otras ciudades, se considera a esta provincia como la máxima aportación a la economía a nivel país puesto que tiene varias organizaciones ya constituidas, sin dejar de lado lo importante en mencionar el puerto lugar que maneja el comercio, con esta premisa en los cantones que están a las afueras de esta provincia se considera que el crecimiento es lento puesto que al ser un lugar 
Bárbara Annabelle Pesántez-Célleri; Juan Carlos Erazo-Álvarez; Cecilia Ivonne Narváez-Zurita; Verónica PaulinaMoreno

pequeño siempre existe la rivalidad entre los negocios y por ende conlleva al fracaso del mismo (Silva, Flores, Montalván \& Alejo, 2018).

En el Cantón Playas Villamil las actividades económicas que sobresalen son la pesca, turismo, y comercio como fuentes de ingresos que solventan al desarrollo económico de la población, con la ventaja de tener una mayor cercanía a la provincia del guayas la visita de los turistas es constante. Sobre las bases de las ideas expuestas es importante fortalecer el turismo comunitario en beneficio de sus habitantes con acciones dirigidas a la ejecución de propósitos establecidos en un determinado tiempo y del entorno que les rodea.

Por tanto el aporte significativo de la investigación se enlaza directamente con el Plan Nacional de Desarrollo 2017-2021 toda una vida, es de vital importancia ya que para incentivar emprendimientos comunitarios se destaca en el objetivo 4 en el Art. 4.3 el cual indica "incentivar el crédito y servicios para la población en el sistema financiero nacional para el desarrollo sostenible, solidario y de igualdad" Secretaría Nacional de Planificación y Desarrollo (SENPLADES, 2017, p.79).

Dentro de ese marco, el problema evidenciado en estas comunidades es la falta de emprendimientos estratégicos, lo que ha causado que las comunidades no puedan salir adelante en los negocios y la duración del mismo sea muy corta, como también la débil instrucción sobre emprendimiento estratégico y la ineficacia en la atención al momento de satisfacer la necesidad del turista; lo que conlleva a los turistas a elegir otras playas de la costa ecuatoriana; ante ello, surge la interrogante de ¿Cómo fortalecer el desarrollo socio-económico de los habitantes del Cantón Playas Villamil?.

Por tanto, al evidenciar ésta problemática se plantea el objetivo de la investigación que es el de diseñar estrategias de emprendimiento de turismo comunitario para fortalecer el desarrollo socio-económico de los habitantes del Cantón Playas Villamil, motivando a la población en la iniciativa de provocar en los turistas que la primera elección como lugar de relajación, esparcimiento, y de inversión sea el cantón, por consiguiente la idea de estrategias de emprendimiento sería importante considerarlas. 
Bárbara Annabelle Pesántez-Célleri; Juan Carlos Erazo-Álvarez; Cecilia Ivonne Narváez-Zurita; Verónica PaulinaMoreno

\section{Referencial teórico}

\section{El desarrollo socio-económico local}

En este sentido, (Aghón, Albuquerque \& Cortés, 2001) abordan el tema de desarrollo socioeconómico, indicando que en base a los recursos con los que cuente la región se puede aprovechar y así elevar el crecimiento económico para la sostenibilidad del lugar. Cabe considerar que en el momento que una comunidad se enfoca en el crecimiento económico de su localidad, van a poder desarrollarse y beneficiarse de los ingresos que puedan alcanzar para el beneficio de su comuna (Erazo-Álvarez \& Narváez-Zurita, 2020).

Dentro de esta perspectiva, (Carvajal-Burbano, 2011) manifiesta que el desarrollo local es más amplio con respecto al desarrollo económico local, ya que esto comprende el crecimiento de un sistema completo que enmarca la extensión del territorio, la económica, la identidad cultural, de gobierno y de ambiente en una región, y esto va de la mano a la expansión de comunidades dirigiéndose a la riqueza de toda la población.

En América latina el desarrollo económico local no se lo minimiza y no se considera que es de poca relevancia, más bien como indica Francisco Albuquerque, citado en (Carvajal-Burbano, 2011), es de gran importancia por cuanto integra todas las acciones de producción que tienen los países, teniendo fundamento esencial para la generación de empleo, la promoción del territorio, proceso técnico y desarrollo económico. Enríquez como se cita en (Carvajal-Burbano, 2011) existen factores que encaminan al crecimiento económico de la población, estos son:

1. Direccionar las gestiones además que en las políticas sociales, en la economía local, incentivando a la creación de trabajo e impulsando el desarrollo regional.

2. Tener disponible la mano de obra capacitada para la realización de actividades, formación empresarial altamente capacitada en la tradición para el beneficio de la localidad. 
Bárbara Annabelle Pesántez-Célleri; Juan Carlos Erazo-Álvarez; Cecilia Ivonne Narváez-Zurita; Verónica PaulinaMoreno

3. Una cultura que tenga un enfoque claro a la modernización y así beneficiar al crecimiento y la preservación del capital en el proceso de producción local.

4. Además de la adquisición de tecnología, también se benefician las localidades en la implementación de innovación para el progreso de la producción.

Así mismo, (Carvajal-Burbano, 2011) indica que el desarrollo económico local ha podido extenderse en las regiones golpeadas por la crisis mundial con respecto al desempleo, el incremento de las decisiones de las políticas de cada país y las circunstancias de cada región que basan su progreso en emprendimientos por falta de empleos. el desarrollo económico local intervienen tanto instituciones públicas, privadas, que están en constante desempeño para aplicar ventajas para el fortalecimiento local y generar empleos para la subsistencia del lugar, la esencia del desarrollo económico local es dar facilidades para el emprendimiento individual dirigido al desarrollo de una región incluso de un país, por medio de desarrollo de ventaja competitiva, los requisitos para poder conformar un marco de política nacional y local, a continuación:

1. Crear y fortalecer las instituciones locales, gestionando el desarrollo económico local.

2. Fortalecer las habilidades en la comunidad.

3. Impulsar el crecimiento de negocios, dando facilidades e incentivando la inversión y creación de nuevos emprendimientos.

4. Publicidad de la competitividad empresarial.

5. Generar ventajas competitivas regionales.

Por otro lado, (Amate-Fortes \& Guarnido-Rueda, 2011), la principal meta del desarrollo económico es permitir que existan situaciones que den un mejor nivel de vida con prosperidad, por tanto debe tener calidad, esta debe conseguirse a través de buenas prácticas con respecto a la política pública para desarrollarse el ámbito social. Según (Carvajal-Burbano, 2011), es de gran importancia apreciar los recursos locales, con una economía sustentada para los actores que intervienen en 
Bárbara Annabelle Pesántez-Célleri; Juan Carlos Erazo-Álvarez; Cecilia Ivonne Narváez-Zurita; Verónica PaulinaMoreno

su crecimiento y tiene un aporte no menor para intervenir a mejoras en distribuir el ingreso local, el propósito es socavar la pobreza, la desigualdad, es necesario volver a plantear enfoques que ayuden a la comunidad, para así promover la generación de riqueza la producción de empleo y acabar con la pobreza de los habitantes.

John Crissien Castillo como se cita en (Martínez-Rodríguez \& Amador-Muñoz, 2013), incita a la creación de un empleo propio para reafiarmar el ámbito empresarial lo cual ayuda al desarrollo en las regiones, éstas se centran en modeladores económicos, en lo político y lo institucional, los cuales ayudan a incentivar la economía, la mejora en los ingresos de los habitantes, por tanto se refiere a que se debe tomar en consideración que está relacioano el desarrollo económico con la educación en los negocios, puesto que al tener educación va a producir un ambiente de prosperidad y crecimiento local (Erazo-Álvarez \& Narváez-Zurita, 2019).

\section{Estrategias de emprendimiento: Concepto e importancia}

El emprendimiento es la iniciación de una idea que aparece frente a necesidades para tener solvencia económica y así satisfacer las mismas, (Schnarch-Kirberg, 2014) comenta que el emprendimiento es incentivar desde la impresión que se tenga con relación al bienestar territorial. El emprendedor crea nuevos negocios, esto a nuevas fuentes de empleo, generando un aumento de mercado de las que se relacionan, una notable mejoría en el rendimiento de las empresas que promueven un cambio tecnológico e innovación y favorecen al escalamiento con respecto a la competitividad entre países.

En concordancia, (Jáuregui \& Carmona, 2014) para llegar a tener triunfo en un negocio es muy sustancial tener cualidades y talentos; Además es aquel que inicia con una decisión arriesgada y difícil, aplicando al entorno empresarial el emprendedor se identifica por tener actitudes decididas y tiende en asumir desafíos, siempre está en busca de la viabilidad de su plan empresarial; el emprendedor para iniciarse en el negocio cuenta con un rol muy definido con respecto a su comportamiento que es el compromiso, persistencia, voluntad, dispuesto a trabajar constantemente, tiene puesta su total confianza en su proyecto, con convicción 
Bárbara Annabelle Pesántez-Célleri; Juan Carlos Erazo-Álvarez; Cecilia Ivonne Narváez-Zurita; Verónica PaulinaMoreno

basada en argumentos fuertes los cuales han sido planificados con anticipación. En la siguiente tabla se expresa de forma más clara las características que tienen los emprendedores y se muestra a continuación:

\section{Tabla 1}

Tipos de emprendedores

Tipos

Emprendedor social

Emprendedor ecológico

Emprendedor artístico y cultural

Emprendedor de negocios

Emprendedor deportivo

Emprendedor Político

\section{Características}

Son aquellos que están preocupados por el beneficio social y desean cambiar su situación crítica

Es aquel que se enfoca en el beneficio ambiental y realiza gestiones que mejoren el ambiente Es aquel emprendedor que busca ser reconocido por sus habilidades artísticas y sacar beneficio del mismo Es aquel que con su visión e iniciativa crea negocios, los cuales generan fuentes de trabajo y rentabilidad para la organización.

Este tipo de emprendedor se motiva para implementar negocio de materiales de uso deportivos, bebidas, etc. generando ganancias al deporte.

Este tipo de emprendedor se relaciona con el emprendedor de negocios ya que los dos tienen una actividad económica en el ámbito que se desenvuelven, el emprendedor político es aquel que genera nuevos decretos legislativos para dar solución a los inconvenientes que tiene la sociedad, y apoya al desarrollo de su bienestar

Fuente: Alcaraz-Rodriguez (2015).

Como se puede observar en la tabla N.-1, en el mundo del emprendimiento existen varios tipos de emprendedores que no solo se basan en tener una organización sino también se desenvuelven en diversos mercados. Por tanto, (Núñez-Ladeveze \& Núñez-Canal, 2016), indican que el emprendimiento es el punto de partida que 
Bárbara Annabelle Pesántez-Célleri; Juan Carlos Erazo-Álvarez; Cecilia Ivonne Narváez-Zurita; Verónica PaulinaMoreno

incentiva a innovar generando cambios para mejoras en el beneficio y capacidad económica.

En relación a lo antes expresado, David McNally como se cita en (PalaciosAcero,2015), enseña que existen 5 cualidades primordiales para los emprendedores, estas son: Quererse a sí mismo, valorarse; Ser visionario sobre: construcción de sueños; Ser objetivo con: objetivos y desafíos; Ser leal con: la salud, familia, el trabajo, la profesión; Contribuir con: Testimonio, creación de valor.

Así mismo, (Palacios-Acero,2015), indica que para tener valor personal en sí mismo y ser un buen emprendedor es necesario tener los siguientes elementos: Autocontrol, autoestima, autoeficacia, autoimagen. Al carecer de uno de estos elementos emerge la inestabilidad emocional en la personalidad, perdiendo objetividad para analizarse y también cambia la manera de ver el entorno. Al emprendedor se lo conoce como inventor, eficiente, flexible, arriesgado, creativo y encaminado a llegar a la cúspide del éxito. Desde un análisis general, con frecuencia se identifica al emprendedor como la capacidad de empezar y manejar compañías nuevas.

Em consecucíon, (Jáuregui \& Carmona, 2014) consideran que para llegar a tener éxito en un negocio es muy sustancial tener actitudes y aptitudes. El emprendedor es aquel que inicia con una decisión arriesgada y difícil, aplicando al entorno empresarial el emprendedor se identifica por tener actitudes decididas y tiende en asumir desafíos, siempre está en busca de la viabilidad de su plan empresarial. El emprendedor para iniciarse en el negocio cuenta con un rol muy definido con respecto a su comportamiento que es el compromiso, persistencia, voluntad, dispuesto a trabajar constantemente, tiene puesta su total confianza en su proyecto, con convicción basada en argumentos fuertes los cuales han sido planificados con anticipación. Las actitudes son vitales para empezar un emprendimiento, y también son las aptitudes adecuadas para emprender.

Las actitudes ya vienen innatas en las personas al contrario de las aptitudes en cambio estas con el tiempo se van formando. Para ser un excelente emprendedor debe lograr tener buena aptitud y actitud antes de arrancar un proyecto a 
Bárbara Annabelle Pesántez-Célleri; Juan Carlos Erazo-Álvarez; Cecilia Ivonne Narváez-Zurita; Verónica PaulinaMoreno

emprender. Por tanto se recomiendan que de acuerdo a lo expuesto anteriormente hay que considerar las siguientes sugerencias para emprender:

1. No tener miedo a fracasar, ya que el fracaso en un proyecto es parte de un proceso de aprendizaje.

2. Instruirse en materia empresarial especialmente en finanzas.

3. Comprender que para emprender no es necesario contar con la más sofisticada tecnología para llegar al éxito.

4. Enfrentar las incertidumbres al inicio ya sean internas o externas para la superación de los desafíos.

5. Usar las plataformas de beneficio a emprendedores que están en municipios, cámaras de comercio, gobierno etc.

6. Relacionarse con más emprendedores que contribuyan al crecimiento.

7. Participar en eventos para buscar reconocimientos a la labor realizada, y adquirir nuevas ideas que ayuden a mejorar el proyecto.

8. Enfocarse en mercados globales.

9. Elaboración de una planificación empresarial y así poder definir la viabilidad del proyecto.

Para (Sastre, 2013), el emprendedor a medida que se fortalece, analiza que el recurso financiero es de vital importancia para mantener el negocio. Con el análisis precedente, considera (Fred, 2013) que la estrategia es el medio por el cual se alcanzan objetivos a un largo tiempo, por lo general se considera como estrategias al desarrollo geográfico, a la obtención, al crecimiento de recursos, la inserción al mercado, la disminución de presupuesto, la desinversión y liquidación en las organizaciones.

De acuerdo a (Fernández-Lorenzo, 2012), por tanto la estrategia es el seguimiento incansable de un proyecto el cual una compañía crea para sostener ventaja competitiva, entendiéndose como ventaja competitiva a la particularidad diferente que tiene cada emprendimiento para llegar a sus fines. Según (Fred, 2013) es importante considerar que las estrategias pueden tener efectos funcionales y 
Bárbara Annabelle Pesántez-Célleri; Juan Carlos Erazo-Álvarez; Cecilia Ivonne Narváez-Zurita; Verónica PaulinaMoreno

divisionales es por esto que al momento de diseñarla se deben considerar aspectos que enfrenta la organización sobre el entorno.

La estrategia según (Cleri, 2016) es la habilidad para realizar acciones que dirigen a la ejecución de propósitos establecidos en un determinado tiempo y del entorno que les rodea. Para (Chiavenato, 2017) la estrategia tiene un fuerte y basto estudio de las acciones con las que cuenta la organización para elegir, partiendo de que sus inicios están basados en una situación con miras a un futuro distinto con intención de llegar a tener utilidades y beneficios con respecto a la actualidad de la empresa.

Al realizar una selección de una estrategia se debe tomar en consideración los aspectos intrínsecos y del exterior, que no llegue a la afectación de la organización. Es por eso que el líder de las estrategias en la empresa siempre considera su elección de estrategia basándose en el cálculo de la pérdida y ganancia que obtendría la empresa por la estrategia elegida, aunque la decisión es basarse dentro de un análisis racional, con la previsión de una probabilidad con respecto a la teoría de juegos (Picón-Vizhñay, Erazo-Álvarez, \& Narváez-Zurita, 2019). Por su parte, para (Koontz \& Weihrich, 2013) consideran que la estrategia global de las empresas puede tener niveles jerárquicos, a continuación se detalla en la Figura 1:

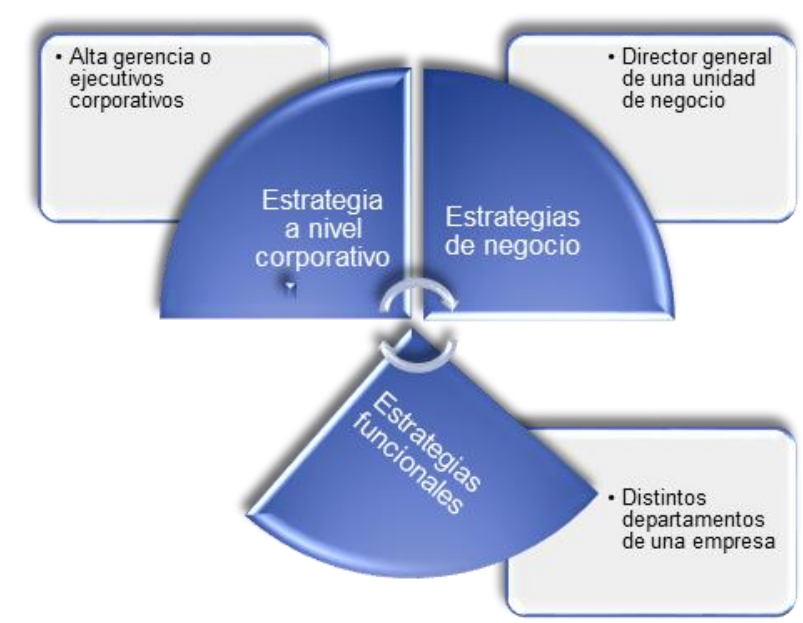

Figura 1. Pirámide de Jerarquías de Estrategias.

Fuente: Koontz y Weihrich (2013). 
Bárbara Annabelle Pesántez-Célleri; Juan Carlos Erazo-Álvarez; Cecilia Ivonne Narváez-Zurita; Verónica PaulinaMoreno

Al inicio de la pirámide consta la estrategia corporativa, es aquí en donde los directivos analizan y forman la estrategia global de la empresa; se realiza la toma de decisión con respecto a la competencia, y se eligen los portafolios que serán los que generen beneficio a la empresa, en la segunda posición de jerarquías, yace la estrategia de negocio, y es realizada por el Gerente General de la compañía, es el director ejecutivo quien acepta o nulita las diferentes estrategias.

Estas estrategias de negocio tienen como finalidad la obtención de ventajas competitivas específicas de alguna línea de producto, indican que ya habiendo llegado a la tercera posición de la pirámide jerárquica es aquí donde se definen estrategias de funciones o de política. Las cuales son estrategias diseñadas para las distintas áreas de la empresa como en lo financiero, productivo, mercadeo, de servicio, y del recurso humano etc. El objeto es dar apoyo a la estrategia del negocio y la corporativa. En consecuencia, (Rodríguez, Taborda, Eula, Camisasso \& Maniaci, 2016) consideran necesario que para crear estrategias hay que fundamentarse en los siguientes elementos para su elaboración:

\section{Tabla 2.}

Elementos fundamentales para la elaboración de estrategias.

\begin{tabular}{ll}
\multicolumn{1}{c}{ Elementos } & \multicolumn{1}{c}{ Descripción } \\
\hline Escenario Situacional & Es el marco de referencia que se \\
sitúa el territorio y la situación a ser \\
analizada. \\
Son los actores que deciden por \\
situaciones que se presentan, de \\
acuerdo al entorno que los rodea. \\
Es la noción que determina una \\
acción dentro de una realidad \\
abjetos & expresada. \\
Son aquellos que están inmersos en \\
varios ámbitos ya sea en lo social, \\
cultural, natural etc. a analizar el \\
Tiempo & $\begin{array}{l}\text { Este se considera al analizan } \\
\text { presente, pasado, para determinar }\end{array}$ \\
acciones correctas. \\
Son la eficacia de las consecuencias \\
Lasuales que contienen sucesos \\
tácticos.
\end{tabular}

Fuente: Rodríguez, Taborda, Eula, Camisasso \& Maniaci (2016). 
Bárbara Annabelle Pesántez-Célleri; Juan Carlos Erazo-Álvarez; Cecilia Ivonne Narváez-Zurita; Verónica PaulinaMoreno

Es importante tener en consideración estos elementos para elaborar estrategias efectivas que sean viables y conlleven al éxito en el ámbito social.

\section{El turismo comunitario}

En este sentido, (Santana-Moncayo \& Atiencia-Hoyos, 2014), exponen a partir de la Oficina Internacional de Trabajo (OIT) que el turismo comunitario es la conformación de personas que se establecen con el sustento de la participación y la preservación del patrimonio comunitario. Enlazando actividades democráticas y de trabajo para atraer beneficios y ofrecer servicios turísticos de calidad para los turistas.

En consideración, (Kekutt, 2014) señala que a través del turismo comunitario se busca aprovechar una mayor productividad y crecimiento es necesario con opciones socio-productivas ya sea para el beneficio de una o algunas comunidades y así poder llegar al fortalecimiento en el aspecto social y de su territorio. El turismo comunitario brinda alternativas de seguridad y desarrollo para su localidad ya que al tener un direccionamiento colectivo brotan mejores ideas para el beneficio y explotación correcta de sus recursos. En la tabla 3, se detallan las formas mas importantes de ejercer gestión turística comunitaria a continuación:

\section{Tabla 3.}

Gestión turística Comunitaria.

Formas de Gestión

\section{Descripción}

La colaboración directa de la comuna Cuando una comunidad emprende es la responsable de manera única del emprendimiento y también lo es toda la comuna, con la dirección del dirigente de la comunidad como persona encargada en promover el turismo.

La colaboración de un grupo de familias nativas con la garantía de su comuna esto ocurre cuando estos grupos de familias son apoyados por la comunidad con ingresos para el emprendimiento, o también se les apoya ya que generan ingresos y beneficios ejerciendo el turismo para toda la comunidad en esta gestión es importante 
Bárbara Annabelle Pesántez-Célleri; Juan Carlos Erazo-Álvarez; Cecilia Ivonne Narváez-Zurita; Verónica PaulinaMoreno

Fuente: Roux (2013).

\begin{abstract}
destacar que todas las comunidades acuerdan en dar servicio turístico ofreciendo cada una un servicio distinto a la otra, como por ejemplo: una comunidad se dedica a realizar tejidos, otra a realizar platos típicos, guías turísticos etc., conocido como turismo particular o de asociación, cabe recalcar que al no tener el respaldo de la comuna, no existe el turismo comunitario, por lo tanto toda actividad debe ser realizada con el fin de aportar al beneficio de la misma comunidad.
\end{abstract}

Se observa claramente en la tabla N.-3 las formas de gestión comunitaria en donde intervienen la participación de las comunas, de sus familias, las alianzas con varias comunidades, (Roux, 2013) considera que existen principios para ejercer turismo comunitario son de vital importancia tener en consideración la preservación del ambiente para ejercer turismo comunitario, a continuación se detallan los siguientes principios:

1. Ambiental.- Es concientizar a la ciudadanía en la protección de los recursos naturales, el patrimonio, la flora, la fauna, de manera que el turismo es el que insta a valorar la naturaleza cuidándola y manteniéndola, evitando que sea contaminada y respetando el control territorial, impidiendo la usurpación de su patrimonio.

2. Cultural.- La labor del turismo exige tener identidad cultural, conserva la lengua nativa, la costumbre de sus antepasados, enseña lo que queda de las comunas, emerge toda práctica, costumbre y el saber tradicional, y ratifica la esencia de la interculturalidad del país.

3. Social.- Por medio del turismo se fomentan las prácticas sobre el derecho social, se enseña la diferenciación entre poblaciones en subdesarrollo y en vías de desarrollo, concientiza a los visitantes con respecto a lo relevante que es el defender el territorio, el turista se instruye por la comunidad y su gente, con el turismo se da a conocer el modus vivendi de las comunidades 
Bárbara Annabelle Pesántez-Célleri; Juan Carlos Erazo-Álvarez; Cecilia Ivonne Narváez-Zurita; Verónica PaulinaMoreno

localmente y en el ámbito internacional, favorece la condición de la comuna, evitando emigrar a otros países, impulsa la unión entre sus comuneros, consolida lazos y genera el cooperativismo, y la consideración entre todos.

4. Económico.- Por el turismo se crean más medios de subsistencia, generación de ingreso, ampliación de la actividad productiva de la comuna, incentiva a la inversión en la instrucción, atención médica, y de otorgar el servicio básico para la comunidad.

5. Político.- El turismo siempre ha ayudado a la comunidad para que sean tomadas en consideración con respecto a sus opiniones e inquietudes, y así lograr su posicionamiento nacional, e identificarse defendiendo sus territorios.

\section{MÉTODO}

La presente investigación se basó en una tipología descriptiva no experimental, representada por el análisis del objeto de estudio (Hernández, Fernández \& Baptista, 2014), indican que este visión se desarrolla a través del uso de variables numéricas, gráficas, fórmulas y modelos analíticos, así se centró en indicar la razón por las variables se comportan en la realidad. Se apoyó en el método analítico-sintético con el cual se realizó un análisis de cómo están las estrategias de emprendimiento en la población de Playas Villamil (Argudo-Tello, Erazo-Álvarez, \& Narváez-Zurita, 2019).

Siendo un trabajo de corte transversal en cuanto a la recopilación de datos en el Cantón Playas Villamil, realizándose en un solo tiempo o momento, mismo que se sustentó de acuerdo a (Martínez-Ruiz \& Benítez-Ontiveros, 2016), mencionan que toda la información extraída es realizada en una sola vez y en un espacio determinado. Por consiguiente se aplicó como técnica de recopilación de información, la encuesta, mediante cuestionario de recopilar datos que posteriormente contribuyeron con el propósito de analizar el comportamiento de las variables de estudio.

Por consiguiente la encuesta estuvo dirigida a los habitantes del Cantón Playas Villamil. Se obtuvo el universo de estudio conformado por: $U=24.496$ habitantes del Cantón Playas Villamil. El método de muestreo que se usó es el probabilístico con 
Bárbara Annabelle Pesántez-Célleri; Juan Carlos Erazo-Álvarez; Cecilia Ivonne Narváez-Zurita; Verónica PaulinaMoreno

la técnica de muestreo estratificado sin reposición, de una población finita; se obtuvo una muestra de 378 habitantes por sexo y entre las edades de 15 años a 59 años, se aplicó nivel de confianza del $95 \% \mathrm{z}=1,96 \quad \mathrm{e}=0.05$, la $\mathrm{p}=0.50 \mathrm{q}=0.50$, a continuación, se detallan datos de información en la tabla 4:

\section{Tabla 4.}

Población por sexo del Cantón Playas Villamil.

$\begin{array}{rrrrrrrrr}15 a & 20 a & 25 a & 30 a & 35 a & 40 a & 45 a & 50 a & 55 a \\ 19 & 24 & 29 & 34 & 39 & 44 & 49 & 54 & 59\end{array}$

Habitantes edades edades edades edades edades edades edades edades edades

\begin{tabular}{llllllllll}
\hline Hombres & 2007 & 1839 & 1768 & 1616 & 1321 & 1194 & 1101 & 881 & 680 \\
Mujeres & 1955 & 1792 & 1723 & 1575 & 1287 & 1163 & 1072 & 859 & 663 \\
\hline
\end{tabular}

Fuente: INEC, (2010).

\section{RESULTADOS}

Mediante la aplicación de los instrumentos de investigación que determinan la problemática evidenciada, se obtuvieron los siguientes resultados: En consideración al turismo comunitario, un $51 \%$ de la población del Cantón Playas Villamil consideran que el turismo comunitario se desarrolla de forma regular, entre tanto un $49 \%$ manifiesta que si tiene un buen desarrollo de turismo comunitario; a su vez, la población considera pertinente en un promedio del $61 \%$ su inclinación hacia negocios con emprendimiento comunitario específicamente en hotelería, artesanías, turismo y gastronomía; no obstante, un $38 \%$ se inclina más por el comercio, la pesca artesanal y actividades agrícolas.

En cuanto a la situación económica laboral de los habitantes del Cantón Playas Villamil un $49 \%$ se divide entre personas desempleadas, estudiantes, jubilados y amas de casa, lo que indica que no cuentan con una actividad establecida para emprender un negocio de emprendimiento comunitario; en tanto un $33 \%$ de ellos forman parte del grupo de empleados perteneciente a empresas o negocios del 
Bárbara Annabelle Pesántez-Célleri; Juan Carlos Erazo-Álvarez; Cecilia Ivonne Narváez-Zurita; Verónica PaulinaMoreno

Cantón, finalmente un porcentaje del $18 \%$ cuentan con negocio propio en actividades diversas dentro del Cantón.

El 74,19\% de los habitantes del Cantón manifiestan que cuentan con conocimientos y habilidades por experiencia para crear un negocio de turismo comunitario y un $25,81 \%$ de ellos consideran que les falta. Un $58 \%$ de los habitantes manifiestan que los empresarios o emprendedores de éxito son reconocidos en el Cantón por la calidad de esfuerzo y trabajo, un $42 \%$ sostiene que los emprendedores no tienen esa motivación. Un $53,60 \%$ de los habitantes estiman que sus necesidades son tomadas en cuenta para la creación de un emprendimiento, y un $46,4 \%$ consideran que no lo son.

Así mismo, un $82 \%$ de ellos estiman que para emprender en turismo comunitario, es importante tener conocimiento técnico sobre emprendimiento, y un $18 \%$ no lo considera necesario. El $67 \%$ de la población indica que la infraestructura les favorece para emprender, y un $33 \%$ manifiestan que no lo considera necesario. El $88 \%$ de los habitantes considera que los turistas buscan productos y servicios innovadores y buenos, entre tanto un $12 \%$ desconocen los productos y servicios innovadores que ofertan a los turistas.

\section{PROPUESTA}

En esta propuesta se da a conocer elementos centrales para el desarrollo de la misma, que se detallan a continuación: Diagnóstico en donde constan las fortalezas, oportunidades, debilidades y amenazas del Cantón, se definen ventajas competitivas, segmentación público objetivo, definición de objetivos y como finalización formulación de estrategias, se detalla en la Figura 2: 
Bárbara Annabelle Pesántez-Célleri; Juan Carlos Erazo-Álvarez; Cecilia Ivonne Narváez-Zurita; Verónica PaulinaMoreno

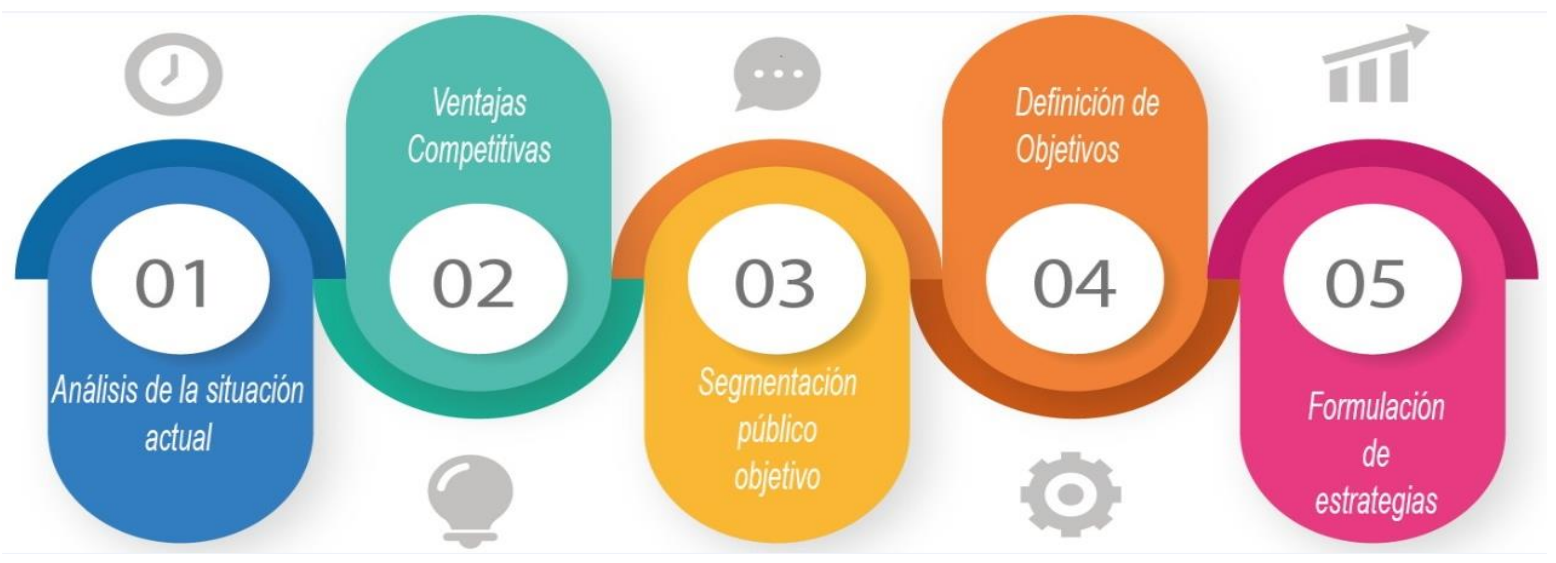

Figura 2. Elementos de la propuesta.

\section{Análisis de la situación actual}

En este punto se describen las debilidades, amenazas, fortalezas y oportunidades del Cantón Playas Villamil, se detalla a continuación:

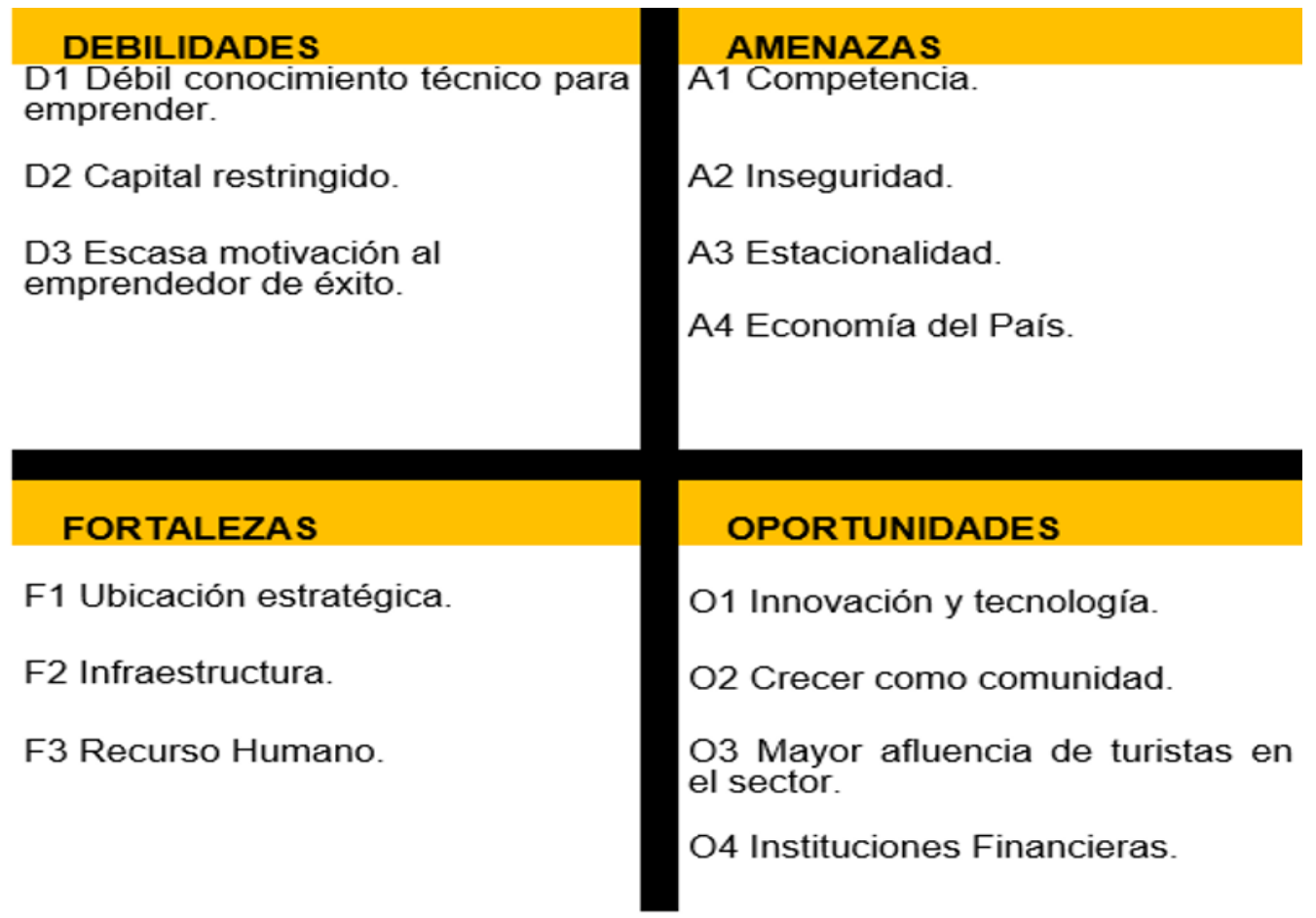

Figura 3. Diagnostico DAFO del Cantón Playas Villamil. 
Bárbara Annabelle Pesántez-Célleri; Juan Carlos Erazo-Álvarez; Cecilia Ivonne Narváez-Zurita; Verónica PaulinaMoreno

En la tabla $\mathrm{N}-5$, se procede a realizar Matriz EFI "Esta herramienta para la formulación de estrategias sintetiza y evalúa las fortalezas y debilidades más importantes encontradas en las áreas funcionales de una empresa y también constituye la base para identificar y evaluar las relaciones entre estas áreas" (Fred, 2013, p.122). En ésta se detallan las debilidades y fortalezas factores internos de la situación actual del Cantón, en el cual mediante ponderación de un semáforo numérico se va clasificando la importancia de cada factor, se observa que la calificación es de 2,70 lo cual quiere decir que al tener mayor calificación en las fortalezas, el Cantón está en la condición de superar las debilidades como a continuación se detalla:

Calificación entre 1 y 4: 4 Fortaleza mayor, 3 Fortaleza Menor, 2 Debilidad Mayor, 1 Debilidad menor.

\section{Tabla 5.}

Evaluación de factores internos, Matriz EFI del Cantón Playas Villamil.

\begin{tabular}{lccc} 
Factores & Peso & Calificación & $\begin{array}{c}\text { Calificación } \\
\text { Ponderada }\end{array}$ \\
Debilidades & $50 \%$ & & \\
\hline iento técnico para emprender. & 0,20 & 3 & 0,60 \\
gido. & 0,136 & 1 & 0,136 \\
ación al emprendedor de éxito & 0,166 & 1 & 0,166
\end{tabular}

Fortalezas $\quad 50 \%$

D1 Débil conocimiento técnic
D2 Capital restringido.
D3 Escasa motivación al
Fortalezas
F1 Ubicación estratégica.
F2 Infraestructura.

D1 Débil conocimiento técnic
D2 Capital restringido.
D3 Escasa motivación al
Fortalezas
F1 Ubicación estratégica.
F2 Infraestructura.

$0,17 \quad 4 \quad 0,68$

F3 Recurso Humano.

0,18

0,146

0,54

Totales

$100 \%$

0,584

Fuente: Elaboración propia a partir de Fred (2013).

De igual manera en la tabla N-6 se procede a realizar el cálculo mediante matriz EFE "Las auditorías externas revelan las oportunidades y amenazas clave a las que se enfrenta la compañía, permitiendo que los gerentes formulen estrategias para aprovechar esas oportunidades y evitar o reducir el impacto de esas amenazas" 
Bárbara Annabelle Pesántez-Célleri; Juan Carlos Erazo-Álvarez; Cecilia Ivonne Narváez-Zurita; Verónica PaulinaMoreno

(Fred, 2013, p.68). Se describen los factores externos para conocer el peso en número y porcentaje ya sea individual o general, en el cual se realiza análisis en donde se obtiene una calificación global de 3.04, esto quiere decir que el Cantón al aprovechar las oportunidades que se presentan puede ir superando las amenazas descritas a continuación se detalla:

Calificación entre 1 y 4: 4 Muy importante, 3 Importante, 2 Poco importante, 1 Nada importante.

\section{Tabla 6.}

Evaluación de factores externos Matriz EFE del Cantón Playas Villamil.

\begin{tabular}{llcc}
\multicolumn{1}{c}{ Factores } & Peso & Calificación & $\begin{array}{c}\text { Calificación } \\
\text { Ponenderada }\end{array}$ \\
\hline A1 Competencia. & $50 \%$ & & \\
A2 Inseguridad. & 0,24 & 3 & 0,72 \\
A3 Estacionalidad. & 0,17 & 2 & 0,34 \\
A4 Economía del País. & 0,09 & 1 & 0,09 \\
$\quad$ Oportunidades & 0,166 & 2 & 0,332 \\
O1 Innovación y tecnología. & $50 \%$ & & 0 \\
O2 Crecer como comunidad. & 0,18 & 3 & 0,54 \\
O3 Mayor afluencia de turistas en el sector. & 0,15 & 2 & 0,30 \\
O4 Instituciones Financieras. & 0,106 & 3 & 0,51 \\
\multicolumn{1}{c}{ Totales } & 2 & 0,212 \\
& $100 \%$ & & 3,044 \\
\hline
\end{tabular}

Fuente: Elaboración propia a partir de Fred (2013).

\section{Ventajas competitivas}

Para definir ventajas competitivas se aplica Matriz Posición estrategia y evaluación de acción (PEYEA) "indica cuáles son las estrategias más adecuadas para una organización determinada: agresivas, conservado-ras, defensivas o competitivas. Los ejes de la matriz PEYEA representan dos dimensiones internas: (la fuerza financiera $[\mathrm{FF}]$ y la ventaja competitiva [VC]) y dos dimensiones externas (la estabilidad del entorno [EE] y la fuerza de la industria [FI]). Es posible que estos cuatro factores constituyan las determinantes más importantes de la posición 
Bárbara Annabelle Pesántez-Célleri; Juan Carlos Erazo-Álvarez; Cecilia Ivonne Narváez-Zurita; Verónica PaulinaMoreno

estratégica general de una organización" (Fred, 2013, p.179). Se describe a continuación en Tabla 7:

\section{Tabla 7.}

Posición estrategia y evaluación de acción, matriz PEYEA del Cantón Playas Villamil.

\section{Fuerzas Financieras}

Solvencia

Apalancamiento

Liquidez

Capital de Trabajo

Riesgos Implícitos del Negocio

Flujos de Efectivo

$$
\text { Valor Promedio }
$$

Fuerzas De La Industria

Abundancia, diversidad de insumos y

proveedores

Potencial de Crecimiento

Conocimientos Tecnológicos

Productividad, aprovechamiento de la capacidad

Demanda

Regulaciones del sector

$$
\text { Valor Promedio }
$$

Ventajas Competitivas

Participación en el mercado

Calidad del producto

Lealtad de los clientes

Control sobre proveedores y distribuidores

Utilización de la capacidad competitiva

$$
\text { Valor Promedio }
$$

Estabilidad Del Entorno

Cambios tecnológicos

Tasa de Inflación

Variabilidad de la demanda

Presión competitiva

Estabilidad política y social

$$
\text { Valor Promedio }
$$

Sumatoria

$$
\text { Eje } X
$$

\section{Calificación}

4

3

3

5

3

4

3,7

Calificación

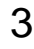

3

4

3

5

4

3,67

Calificación

$-4$

$-5$

$-3$

$-4$

$-2$

$-3,6$

Calificación

$-5$

$-2$

$-4$

$-4$

$-5$

$-4$

Sumatoria

Eje $Y$

0,10

Fuente: Fred (2013). 
Bárbara Annabelle Pesántez-Célleri; Juan Carlos Erazo-Álvarez; Cecilia Ivonne Narváez-Zurita; Verónica PaulinaMoreno

Se procede a plasmar la sumatoria de $(X)$ y $(Y)$ ubicándose en el cuadrante superior izquierdo con los resultados $(-0,33$ y 0,10$)$ teniendo como decisión aplicar estrategias conservadoras, detallado a continuación en la figura 4 :

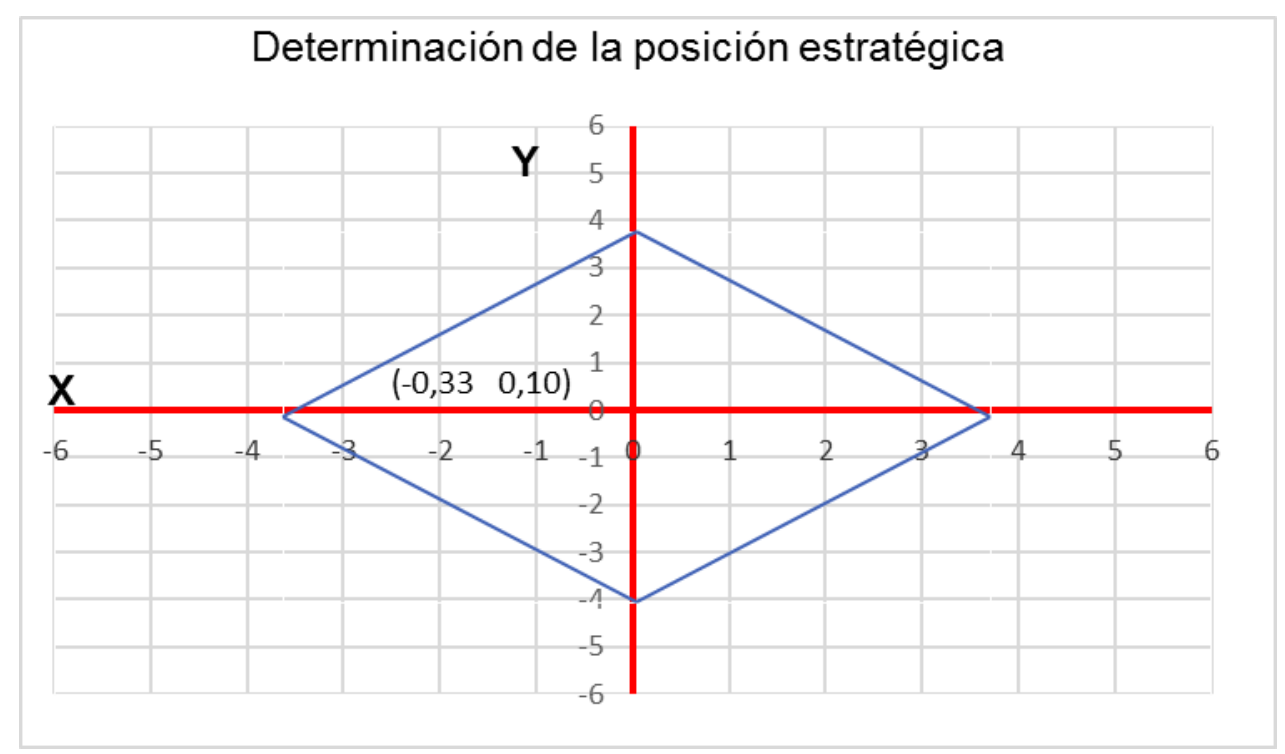

Figura 4. Determinación de la posición estratégica.

\section{Segmentación público objetivo}

Para la segmentación se detallan los aspectos cuantitativos y cualitativos en la siguiente tabla 8:

\section{Tabla 8}

Segmentación del público objetivo

\begin{tabular}{lll}
$\begin{array}{l}\text { Variables de } \\
\text { segmentación }\end{array}$ & $\begin{array}{l}\text { Aspectos cuantitativos en } \\
\text { general }\end{array}$ & $\begin{array}{l}\text { Aspectos cualitativos en } \\
\text { general }\end{array}$ \\
\hline Geográfica & $\begin{array}{l}\text { Público objetivo: } \\
\text { habitantes del Cantón }\end{array}$ & $\begin{array}{l}\text { En base a las } \\
\text { metodologías de análisis }\end{array}$ \\
Pemográfica & $\begin{array}{l}\text { Playas Villamil. } \\
\text { Edades: entre 15 a } 59\end{array}$ & En base a las \\
& años. & metodologías de análisis \\
& Sexo: Masculino y & \\
& Femenino & \\
\hline
\end{tabular}


Bárbara Annabelle Pesántez-Célleri; Juan Carlos Erazo-Álvarez; Cecilia Ivonne Narváez-Zurita; Verónica PaulinaMoreno

\section{Definición de objetivos}

Para definir los objetivos se toma como referencia la identificación de los criterios que están contenidos en la "estrategia conservadora los cuales son: Penetrar en el mercado, desarrollar el mercado, desarrollar el producto y diversificación concéntrica" (Fred, 2013, p.178). A continuación se expresan los objetivos:

1. Motivar a los habitantes del Cantón Playas Villamil al desarrollo de un emprendimiento.

2. Conocer la intensión de emprendimiento de los habitantes del Cantón Playas Villamil.

3. Fomentar la colaboración en las promociones hacia el turista.

4. Incentivar al desempeño de éxito en los emprendedores.

\section{Formulación de Estrategias}

Se desarrollan las distintas estrategias, las cuales se dan a conocer a continuación en tabla 9:

\section{Tabla 9.}

Formulación de estrategias.

\begin{tabular}{|c|c|c|c|}
\hline Nudo crítico & ¿Qué hacer? & ¿Quién hace? & $\begin{array}{c}\text { ¿Quién } \\
\text { coopera? }\end{array}$ \\
\hline $\begin{array}{l}\text { Débil instrucción } \\
\text { sobre } \\
\text { emprendimiento } \\
\text { estratégico }\end{array}$ & $\begin{array}{l}\text { - Incentivar a } \\
\text { talleres } \\
\text { constantes } \\
\text { sobre } \\
\text { emprendimiento } \\
\text { y turismo, } \\
\text { consensuado } \\
\text { con los líderes } \\
\text { locales, } \\
\text { considerando y } \\
\text { los permisos } \\
\text { respectivos. }\end{array}$ & $\begin{array}{ll}\text { a } & \begin{array}{l}\text { Dirigente } \\
\text { Barrial }\end{array} \\
\text { /Presidente/a } \\
\text { de } \\
\text { de Asociaciones } \\
\text {, del Cantón. } \\
\text { s } \\
\text { y } \\
\text { s }\end{array}$ & $\begin{array}{lr}\begin{array}{l}\text { Solicitar } \\
\text { apoyo }\end{array} & \text { el } \\
\text { Director/a } & \text { del } \\
\text { Municipal } & \text { de } \\
\text { Turismo } & \text { GAD } \\
\text { de Playas. }\end{array}$ \\
\hline
\end{tabular}

Escasas actividades relacionadas con emprendimientos
- Potenciar ferias artesanales, gastronómicas con
Dirigente
Barrial /Presidente/a de

Solicitar el - apoyo director/a municipal de 
Bárbara Annabelle Pesántez-Célleri; Juan Carlos Erazo-Álvarez; Cecilia Ivonne Narváez-Zurita; Verónica PaulinaMoreno

estratégicos

\section{Escasa}

promoción de estrategias social media sobre emprendimiento

\section{Escasa} promoción motivación emprendedor comunitario. novedosos sin perder las raíces del Cantón, con los permisos de las autoridades locales.

- Potenciar eventos constantes comunitarios de recreación todo público por ejemplo desfiles trajes típicos con los permisos de las autoridades locales.

- Promoción de emprendimiento $S$ por medio de redes sociales

Asociaciones del Cantón.

Turismo GAD de Playas.
- Promocionar por medio de Guía virtual turística los lugares a visitar en el Cantón Playas Villamil.

- Potenciar a los emprendedores mediante las redes sociales difundiendo actividades y las buenas

prácticas de negocio.

- Crear un fondo de estrategias de innovación que impulsen el desarrollo de nuevas plazas de empleo respetando su originalidad.

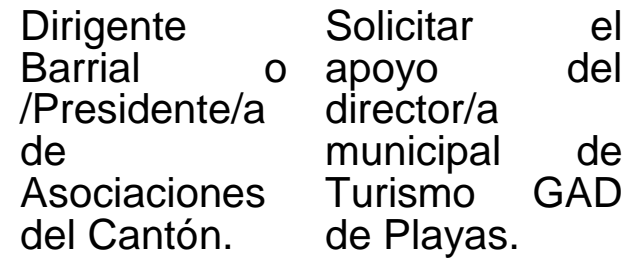

Dirigente Solicitar el Barrial 0 apoyo del /Presidente/a director/a de Municipal de Asociaciones Turismo GAD del Cantón. de Playas. 
Bárbara Annabelle Pesántez-Célleri; Juan Carlos Erazo-Álvarez; Cecilia Ivonne Narváez-Zurita; Verónica PaulinaMoreno

\section{CONCLUSIONES}

En el Cantón Playas Villamil se identificaron factores que influyen en los emprendimientos comunitarios, como la falta de capacitación en áreas relacionadas a estrategia. El diseño de estrategias de emprendimiento de turismo comunitario servirán para fortalecer el desarrollo socio-económico de los habitantes del Cantón Playas Villamil; con la aplicación de matriz DAFO, se describe la situación actual del Cantón en relación a emprendimiento.

Con la matriz PEYEA mediante el análisis de las fuerzas financieras, fuerzas de la industria, ventaja competitiva, y la estabilidad del entorno se identificó la estrategia conservadora; además, se aplicaron las matrices EFE y EFI, por medio de las cuales se analizaron los factores externos e internos del Cantón y ayudaron a tener una perspectiva más clara para determinar la formulación de estrategias; además existen factores que contribuyen al crecimiento de la comunidad tales como: ubicación estratégica, infraestructura, recurso humano, innovación y tecnología, sin olvidar las debilidades y amenazas a las que se enfrenta. Es necesario considerar la motivación a los emprendedores para dar a conocer sus habilidades, mediante la aplicación de herramientas social media, para de esta manera desarrollar emprendimientos estratégicos que tendrían un mejor desenvolvimiento comunitario en el Cantón.

\section{FINANCIAMIENTO}

No monetario.

\section{AGRADECIMIENTO}

A los habitantes del Cantón Playas Villamil, quienes apoyaron el desarrollo de la Investigación con pertinencia social. 
Bárbara Annabelle Pesántez-Célleri; Juan Carlos Erazo-Álvarez; Cecilia Ivonne Narváez-Zurita; Verónica PaulinaMoreno

\section{REFERENCIAS CONSULTADAS}

Aghón, G., Albuquerque, F., \& Cortés, P. (2001). Desarrollo Económico local y descentralización en América Latina: Análisis comparativo. [Local Economic Development and Decentralization in Latin America: Comparative Analysis]. Recuperado de https://n9.cl/ksuj

Alcaraz-Rodriguez, R. (2015). El emprendedor de éxito. [The successful entrepreneur]. México: Mc Graw Hill.

Amate-Fortes, I., \& Guarnido-Rueda, A. (2011). Factores determinantes del desarrollo económico y social. [Determinants of economic and social development]. Recuperado de https://n9.cl/0l9j

Argudo-Tello, K. J., Erazo-Álvarez, J. C., \& Narváez-Zurita, C. I. (2019). Evaluación de Control Interno en Riesgos Estratégicos para la Dirección de Planificación de la Universidad de Cuenca. [Evaluation of Internal Control in Strategic Risks for the Planning Direction at Cuenca University.]. Revista Arbitrada Interdisciplinaria Koinonía, 1 (4), 67-96. http://dx.doi.org/10.35381/r.k.v4i1.372

Carvajal-Burbano, A. (2011). Desarrollo local: manual básico para agentes de desarrollo local y otros actores. [Local development: basic manual for local development agents and other actors]. Recuperado de https://n9.cl/hyuq

Chiavenato, I. (2017). Planeación estratégica, Fundamentos y aplicaciones. [Strategic planning, Fundamentals and applications] (Tercera ed.). (P. Obón, Trad.) México: Mc Graw Hill.

Cleri, C. (2016). Estrategia Bonsai. [Bonsai Strategy]. Buenos Aires: Ediciones Granica Argentina.

Erazo-Álvarez, J. C., \& Narváez-Zurita, C. I. (2019). Conocimiento, capital intelectual y desarrollo como procesos sociales en el clúster cuero y calzado de la provincia de Tungurahua, Ecuador. [Knowledge, intellectual capital and development as social processes in the leather and footwear cluster in Tungurahua province, Ecuador.]. Polo de conocimiento, 9(11), 85-121.

Erazo-Álvarez, J. C., \& Narváez-Zurita, C. I. (2020). Medición y gestión del capital intelectual en la industria del cuero - calzado en Ecuador. [Measurement and management of intellectual capital in the leather industry - footwear in Ecuador]. Revista Arbitrada Interdisciplinaria Koinonía, 9(5), 437-467. http://dx.doi.org/10.35381/r.k.v5i9.662

Fernández-Lorenzo, A. (2012). Metodología para elaborar Planes de Negocio: Análisis a realizar y definición de Modelos de Negocio. [Methodology to develop Business Plans: Analysis to be carried out and definition of Business Models]. Recuperado de https://n9.cl/61dpz 
Bárbara Annabelle Pesántez-Célleri; Juan Carlos Erazo-Álvarez; Cecilia Ivonne Narváez-Zurita; Verónica PaulinaMoreno

Fred R, D. (2013). Conceptos de administración estratégica [Strategic management concepts] (Decimocuarta ed.). Mèxico: Pearson Educación.

Hernández, R., Fernández, C., \& Baptista, M. (2014). Metodología de la Investigación. [Research Methodology]. Mexico DF: McGraw-Hill / Interamericana Editores, S.A. DE C.V.

Institudo Nacional de Estadística y Censos (2019). Encuesta Nacional de empleo, desempleo y subempleo. [National Survey of employment, unemployment and underemployment]. Recuperado de https://n9.cl/nd0u

Institudo Nacional de Estadística y Censos (2010). Población por grupos de edad, según provincia, cantón, parroquia y área de empadronamiento. [Population by age groups, according to province, canton, parish and enumeration area] recuperado de https://n9.cl/j10b

Instituto nacional de estadística y censos (2010). Población por sexo, según provincia, parroquia y cantón de empadronamiento. [Population by sex, by province, parish and canton of enumeration]. Recuperado de https://n9.cl/cdh4

Jáuregui, F., \& Carmona, L. (2014). 1001 Consejos para emprender. [1001 Tips for Entrepreneurship]. Recuperado de https://n9.cl/2yzwh

Kekutt, E. B. (2014). Turismo: Herramienta Social. [Tourism: Social Tool.]. Recuperdo de https://n9.cl/9may

Koontz, H., \& Weihrich, H. (2013). Elementos de Administración. [Management Elements]. (Octava ed.). México: Mc Graw Hill.

Martínez-Rodríguez, F., \& Amador-Muñoz, L. (2013). Educación y desarrollo socioeconómico. [Socio-economic education and development]. Contextos Educativos. Revista de Educación, O(13), 83-98. https://doi.org/10.18172/con.628

Martínez-Ruiz, H., \& Benítez-Ontiveros, L. (2016). Metodología de la investigación social I. [Social Research Methodology I]. Recuperado de https://n9.cl/nmiu

Núñez-Ladeveze, L., \& Núñez-Canal, M. (2016). Noción de emprendimiento para una formación escolar en competencia emprendedora. [Notion of entrepreneurship for school training in entrepreneurial competence.]. Revista Latina de Comunicación Social, (71), 1069-1089. Recuperado de https://n9.cl/fm4p

Palacios-Acero, L. C. (2015). Estrategias de creación empresarial. [Business creation strategies]. Recuperado de https://n9.cl/cleu 
Bárbara Annabelle Pesántez-Célleri; Juan Carlos Erazo-Álvarez; Cecilia Ivonne Narváez-Zurita; Verónica PaulinaMoreno

Picón-Vizhñay, J., Erazo-Álvarez, J., \& Narváez-Zurita, C. (2019). Plan de expansión empresarial para la Empresa Transnexos del Grupo Industrial Graiman. [Business expansion plan for the Transnexus Company of the Graiman Industrial Group]. Revista Arbitrada Interdisciplinaria Koinonía, 1(4), 274-308. http://dx.doi.org/10.35381/r.k.v4i1.459

Rodriguez, E. M., Taborda M., A., Eula, M. L., Camisasso, M., \& Maniaci, A. (2016). Planificación Estratégica: Fundamentos y herramientas de actuación [Strategic Planning: Fundamentals and tools for action]. Recuperado de https://n9.cl/y6v8

Roux, F. (2013). Turismo comunitario Ecuatoriano, conservación ambiental y defensa de los territorios [Ecuadorian community tourism, environmental conservation and defense of the territories]. Recuperado de https://n9.cl/6fOu

Santana-Moncayo, C. A., \& Atiencia-Hoyos, M. C. (2014). Turismo comunitario reflexiones. [Community tourism reflections]. Recuperado de https://n9.cl/kdep

Sastre, R. (2013). La motivación emprendedora y los factores que contribuyen con el éxito del emprendimiento. [Entrepreneurial motivation and factors that contribute to the success of entrepreneurship]. Recuperdo de https://n9.cl/vrw6

Schnarch-Kirberg, A. (2014). Emprendimiento Exitoso. [Successful Entrepreneurship]. Recuperado de https://n9.cl/6so8

Secretaría Nacional de Planificación y Desarrollo, SENPLADES (2017). Plan Nacional de desarrollo 2017- 2021 toda una vida. [National Development Plan 2017-202. A lifetime]. Recuperado de https://n9.cl/znx4

Silva-Guerrero, B. E., Flores-Villacrés, E. J., Montalván-Espinoza, J. A., \& AlejoMachado, O. J. (2018). Propuesta metodológica para el desarrollo de las pymes en la ciudad de Guayaquil Ecuador. [Methodological proposal for the development of SMEs in the city of Guayaquil, Ecuador]. Revista Espacios, 39(42), 12. Recuperado de https://n9.cl/ealw 